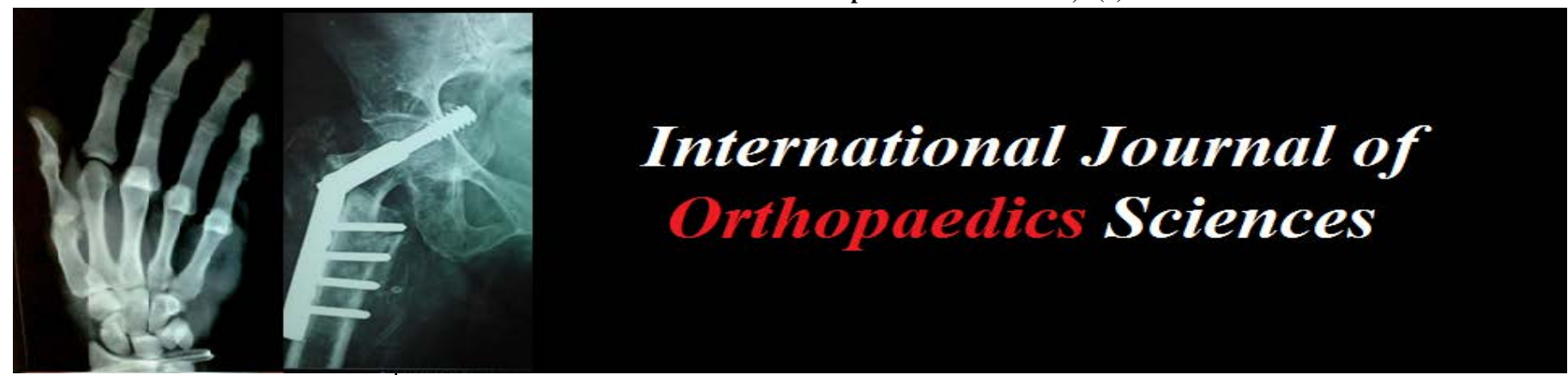

E-ISSN: 2395-1958

P-ISSN: 2706-6630

IJOS 2020; 6(1): 53-57

(C) 2020 IJOS

www.orthopaper.com

Received: 06-11-2019

Accepted: 10-12-2019

Dr. Eesari Kalyan Kuma Junior Resident, Department of Orthopaedics Mamata Medical College, Khammam, Telangana, India

Dr. Abdul Bari

Assistant Professor, Department of orthopaedics, Mamata medica College, Khammam, Telangana, India
Corresponding Author: Dr. Abdul Bari Assistant professor, Department of Orthopaedics, Mamata medical College, Khammam, Telangana, India

\section{A comparative study of clinical outcome of surgical management of supracondylar fracture of humerus in children with crossed (Medial and Lateral) $k$-wire fixation and two lateral $k$-wire fixation}

\author{
Dr. Eesari Kalyan Kumar and Dr. Abdul Bari
}

DOI: https://doi.org/10.22271/ortho.2020.v6.i1b.1835

\section{Abstract}

Supracondylar fractures of Humerus comprise about 17 percent of all childhood fractures. Treatment of supracondylar fractures has included closed reduction and casting in hyper- flexion, traction, open reduction with pinning and closed reduction with pinning. The goal of all forms of treatment is the same, to obtain and maintain an anatomic reduction of the distal humerus to minimize complications such as nerve injury, compartment syndrome.

Volkmann ischaemic contracture, Cubitus varus deformity and limitation of elbow movements. The nonoperative management of supracondylar fracture of humerus including skin traction, skeletal traction and cast application has historically been associated with a greater incidence of failure to obtain and maintain the fracture reduction and subsequent complications as compared with surgical line of treatment. The high rate of complications associated with non-operative treatment led to the evolution of current techniques of percutaneous pinning for these difficult fractures over the past three decades.

Standardization of surgical techniques for performing pin fixation with radiographic control has markedly reduced the incidence of poor outcomes. The advantages of percutaneous pinning methods include easier management of extensively swollen elbows, better maintenance of reduction and decreased risk of associated complications. 2 The present study is an attempt towards assessing and comparing the results of two methods of pinning-crossed pinning and lateral pinning - presently followed in the management of these difficult fractures.

Keywords: Humerus, supracondylar region, kirschner wires

\section{Introduction}

Aims and objectives

1. To study the effectiveness of cross pinning and lateral pinning in treating supracondylar fracture of humerus surgically.

2. To study the outcome of supracondylar fracture of humerus by $\mathrm{k}$ wire fixation individually.

3. To compare the outcome of crossed $\mathrm{k}$ wire fixation and two lateral $\mathrm{k}$ wire fixation.

\section{Complications of supracondylar fracture of Humerus}

In supracondylar fracture of humerus the complications are divided into.

1. Immediate

2. Delayed or late complications the immediate complications are

1. vascular complications

2. Neurological complication.

The delayed complications are

a. Malunion leading to Cubitus varus or Valgus deformity.

b. Myositis ossificans.

c. Stiffness of elbow

d. Infection. 


\section{Vascular Complications}

Vascular complications occurring with extension type of supracondylar fractures are the most serious sequelae of any fracture seen in the paediatric age group. The outcome ranges from fibrosis of muscles to gangrene and subsequent amputation.

The vascular injury may range from a simple compression of the brachial artery to a complete rupture by the fracture fragments. The secondary effects from ischaemia are divided into 4 stages of severity by Ottolenghi 49.

1. Massive gangrene and peripheral gangrene (Volkmann ${ }^{\text {ee }} \mathrm{s}$ contracture).

2. Partial muscle necrosis.

3. Ischaemic fibrosis.

4. Intermittent claudication where deficient circulation is not apparent at rest but present with activity.

Ottolenghies series presents convincing evidence that prompt exploration of the arterial structures can markedly decrease the dreaded, complication of Volkmann ${ }^{\text {ee }}$ contracture

\section{Neurological Complications}

In Wilson "s series of 4520 fractures it is $7 \%$. Radial nerve is the most common. In 1970, Hardegon found that the presence of posteriomedial displacement of the distal fragment was related to radial nerve injury, conversely posteriolateral displacement of the distal fragment is associated with median nerve injury, and often brachial artery involvement. Ulnar nerve injuries are uncommon with extension type of supracondylar fractures.

Recovery of radial nerve palsy has been near total. With median nerve, motor recovery occurred by 7-12 weeks. In ulnar nerve injury sensation returned before motor function in a time range of 19 days to 30 weeks.

\section{Late Complications}

1. Elbow stiffness: Loss of mobility for practical purposes, functional loss of motion is not a problem with extension type of supracondylar fracture

2. Myositis ossificans: Myositis ossification is rare; it is common in fracture and injuries around elbow treated by traditional bone setters.

3. Angular deformities: The angular deformities that can develop after supracondylar fractures are primarily in either the sagittal or coronal plane. In the coronal plane, the deformities are manifest as Cubitus varus or valgus.

4. In the sagital plane, the deformity is manifest as loss of flexion or extension. The Cubitus varus deformity in the most common and produces the greatest concern.

\section{Surgical Methods \\ Crossed Pinning}

When crossed pinning is employed, Zaltz et al. 54 advocated that it is safer to insert lateral pin first, so that the medial pin can be inserted with the elbow in less flexion. This will reduce the tension on the ulnar nerve and allow it to fall posteriorly.55 on the lateral side, the pinpoint was moved about gently under the skin until it was engaged against lateral epicondyle. The pin was then directed upwards and medially at an angle of 35 - 40 degrees to the sagittal plane and 10 degrees posterior to the coronal plane of the humerus. Thus the pin is passed through 35 the distal fragment and the medullary cavity of the proximal fragment to engage the farther cortex of the proximal fragment about $3 \mathrm{cms}$ above the fracture line.

After a provisional fracture stability is obtained with lateral pinning, the medial pin was inserted through the centre of the medial epicondyle in a similar manner with extension of elbow.

Hyperflexion of the elbow is avoided while inserting the medial pin, so that the ulnar nerve in the ulnar groove can be easily avoided by pushing it posteriorly. As the pins are inserted, a resistance is appreciated as the pin traverses through the opposite cortex of the proximal fragment.

\section{Lateral Pinning}

When two parallel or slightly divergent lateral „K $\mathrm{K}^{\text {ce }}$ wires are used, one of the lateral pins generally engages the ossfied centre of the capitellum and passes proximally up the lateral column and engages the medial cortex of humerus at least $1 \mathrm{~cm}$ above the fracture site. The second pin is then introduced parallel or in a slightly divergent position to the first one. Care is taken to maintain approximately $1 \mathrm{~cm}$ spread between the pins and to avoid pins crossing at the fracture site to maintain maximal construct strength. Once the pins are in place, the elbow is extended and the adequacy of reduction is assessed with AP and lateral images.

After leaving about $1 \mathrm{~cm}$ of the pins outside the skin, pins are cut off and bent and a well-padded posterior above elbow slab is applied with elbow flexed to 90 degrees or less as tolerated. Open reduction and internal fixation for displaced (Type III and Type lV) supracondylar fracture of humerus, four types of surgical approaches are described for open reduction and internal fixation with $\mathrm{K}$-wires. This surgical management should be undertaken within 5 to 6 days of injury otherwise the chances of development of myositis ossificans are more.

1. Campbell's posterior approach.

2. Antero- medial approach.

3. Antero -lateral approach.

4. Medial- lateral approach.

The posterior approach is most commonly used in many countries even though it is blamed that this approach affects the vascularity to the elbow and cause for post-operative stiffness of elbow

The K-wires are usually removed at 3 to 4 weeks after taking the check $\mathrm{x}$-rays to confirm the union. Then elbow is supported in a sling and cuff with advice of active movement but weight bearing activities of upper limbs must be avoided up to 8 weeks.

The treatment of flexion type of supracondylar fracture of humerus is differs from that of extension type. The incidence is 2 to $8 \%$ of all supracondylar fracture of humerus. Most of the reports relate to closed reduction and extension cast application. There is little in the literature about surgical management of this flexion type of supracondylar fractures. 
CASE 1
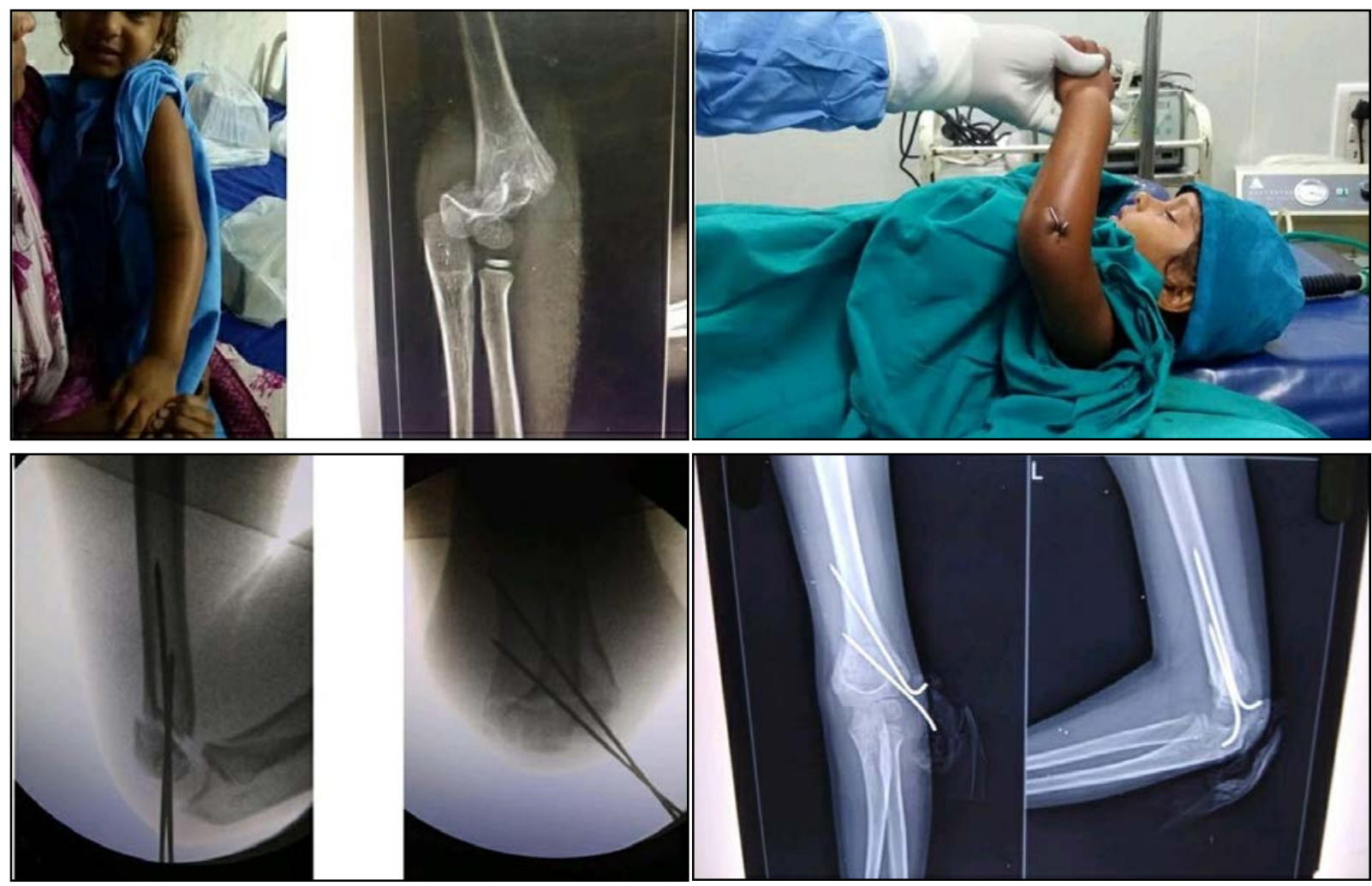

Fig 1: Surgery hand of the joint

\section{CASE 2}

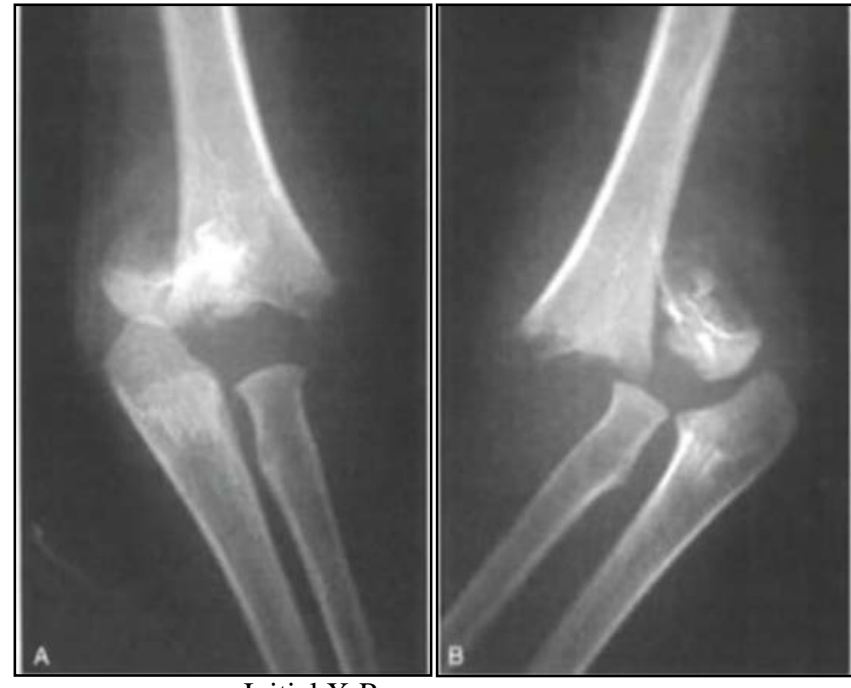

Initial X-Rays
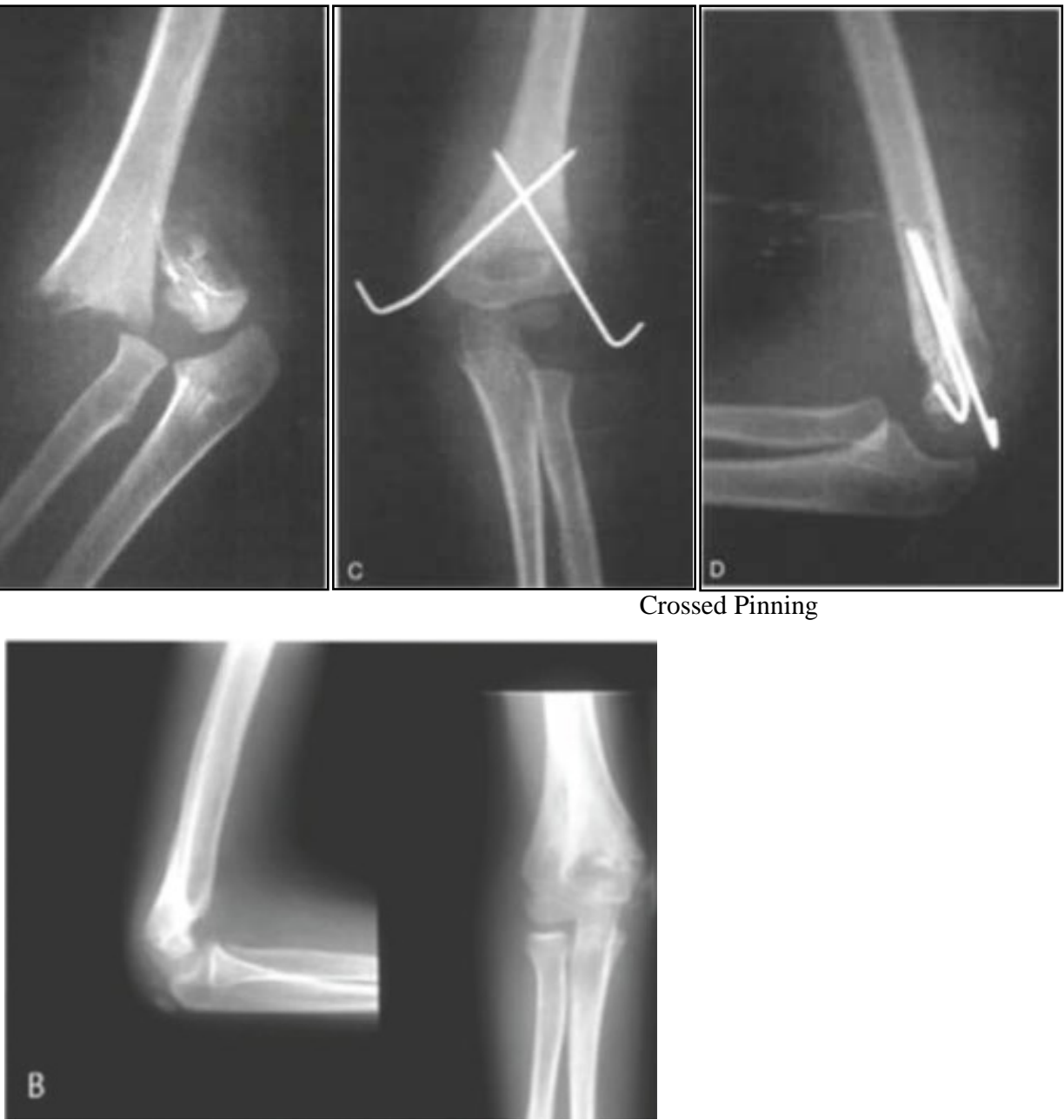

Fig 2: Follow-Up 


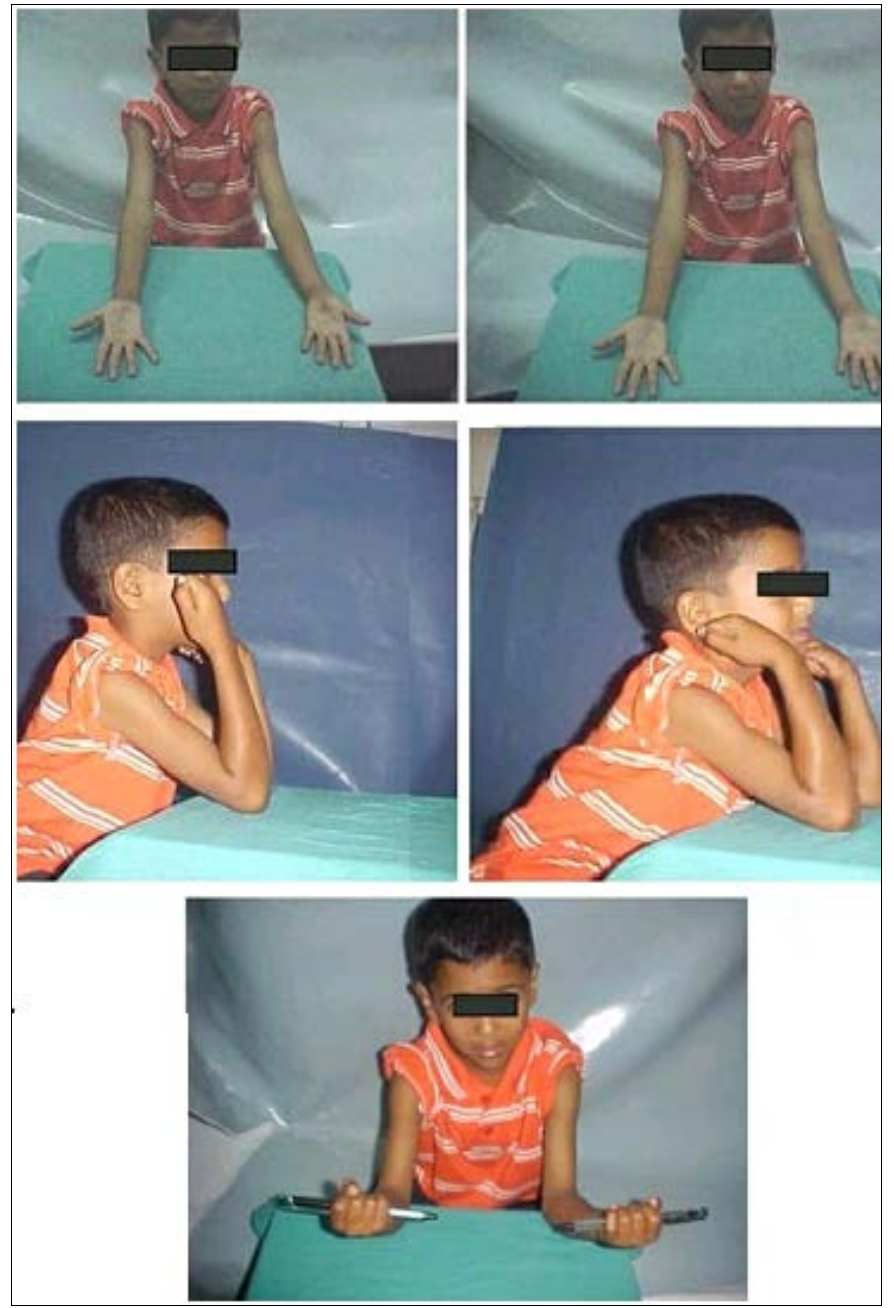

Fig 3: Clinical Pictures in Post Op Review

\section{Discussion}

Supracondylar fracture of humerus is a common fracture seen in children. As its management poses a number of problems like VIC, Nerve palsies, myositis ossificans or Cubitus varus or valgus deformity. It has to be managed with minimal manipulation, anatomical reduction, and fixation to obtain excellent results. Alignment of distal humeral fragment can be achieved either by static means such as K- wire fixation or dynamically by traction. But the disadvantage of traction is long stay in hospital with close observation and increase in recurvatum of elbow. Open reduction and internal fixation, having advantage but the main complication of OR+IF are infection, stiffness of elbow, and more hospital stay.

In these fractures closed reduction and percutaneous pinning have better advantages when compared to all other procedures like its takes less intra operative time, better fixation, and post operatively less complications like infections, elbow stiffness and less hospital stay. In this study, fortyfive children with supracondylar fractures of humerus who were treated with closed reduction and percutaneous crossed pinning (mediallateral) or lateral pinning methods were evaluated both retrospectively and prospectively.

\section{Conclusion}

In our study, we observed that closed reduction and percutaneous pinning is an excellent method of treatment of supracondylar fractures in children. Crossed medial and lateral pinning is the treatment of choice in these fractures, with careful technique which safeguards the ulnar nerve.

We also observed that the lateral pinning is an equally good treatment choice especially for the grossly swollen elbows in which the medial epicondyle in barely palpable with increased risk of ulnar nerve injury during the placement of the medial pin. Both the methods offer consistently satisfactory functional and cosmetic results.

\section{References}

1. Smith HC. Position in the treatment of elbow joint fractures: an experimental study Boston Med. Surgeons J. 1894; 131:386.

2. Poland J. Traumatic Separation of Epiphysis. London, Smith, Elder \& Co, 1898.

3. Bohler L. Technik der Knochenbruchbehandlung. Vienna, Mandrich, 1930.

4. Mac Lemman A. Common fractures about the elbow in children Surg. Gyneco. Obstet. 1947; 64:447.

5. Attenborough CG. Remodelling of the Humerus after Supracondylar Fractures in Childhood. J Bone Joint Surg. 1953; 35:386-359.

6. Madsen E. Supracondylar Fractures of the Humerus in Children. J Bone Joint Surg. 1955; 37:241-245.

7. Gartland JJ. Management of Supracondylar Fractures of the Humerus in Children. Surg. Gynecol. Obstet. 1959; 109:145-154.

8. French PR. Varus Deformity of Elbow Following Supracondylar Fractures of the Humerus in Children. Lancet. 1959; 2:439-441.

9. Mitchell WJ, Adams JP. Supracondylar Fractures of the Humerus in Children. JAMA. 1961; 175:573-577, 76.

10. Gruber MA, Hudson OC. Supracondylar Fracture of the 
Humerus in Childhood. J Bone Joint Surg. 1964; 46:1245.

11. Ramesy R, Hand Griz J. Immeate open reduction and internal fixation of severely displaced supracondylar fixation of severely displaced supracondylar fractures of humerus in children Chin. Orthop. 1973; 90:130-132.

12. D Ambrosia RD. Supracondylar Fractures of Humerus: Prevention of Cubitus Varus. J Bone Joint Surg. 1972; 54:60-66.

13. Dodge HS. Displaced Supracondylar Fractures of the Humerus in Children: Treatment by Dunlopes Traction. J Bone Joint Surg. 1972; 54:1408-1418.

14. Allonso-Llamas M. Billatero tricipital approach to the elbow ActOrthop Scand. 1972; 43:479-490.

15. Ramsey RH, Griz J. Immediate open reduction and internal fixation of severely displaced supracondylar fractures of the humerus in children. Clin. Orthop. 1973; 90:130.

16. Fowles JV, Kassab MT. Displaced Supracondylar Fractures of the Elbow in Children. J Bone Joint Surg. 1974; 56:490-500, 77.

17. Flynn JC, Matthews JG, Benoit RL. Blind pinning of Displaced Supracondylar Fractures of the Humerus in Children. J Bone Joint Surg. 1974; 56:263-273.

18. Arino VL, Lluch EE, Ramirez RM et al. Percutaneous Fixation of Supracondylar Fractures of the Humerus in Children. J Bone Joint Surg. 1977; 59:914-916.

19. Gjerloff C, Sojbjerg JO. Percutaneous pinning of the supracondylar fractures of the Humerus. Acta Orthop. Scand. 1978; 49:597-599.

20. Prietto CA. Supracondylar Fractures of the Humerus. J Bone Joint Surg. 1979; 61:425-428. 